\title{
Psychogenic Coma in Older Adult with Major Depressive Disorder
}

\author{
Saleha Al-Jadidi ${ }^{1,2,3}$, Nooh Al-Harthi ${ }^{1}$ \\ ${ }^{1}$ Al Masarra Hospital, Ministry of Health, Oman; ${ }^{2}$ Oman Medical Specialty Board; ${ }^{3}$ SJMC, Mind and Body Clinic, Oman.
}

\begin{abstract}
Corresponding Author:
Dr Saleha Al-Jadidi

Email: aljadidi_s@hotmail.com

This is an Open Access article distributed under the terms of the Creative Commons Attribution License (creativecommons.org/ licenses/by/3.0).
\end{abstract}

Received

Accepted

July 19, 2020

Published

\begin{abstract}
Background: Coma is defined as lack of both awareness and response to external stimulation. Psychogenic coma is unresponsiveness without organic causes, it described in DSM IV as dissociative disorder NOS. Case Report: This case report is first psychogenic coma in older adult with major depressive disorder reported in Oman. Conclusion: Management of a coma due to dissociative disorder NOS is largely supportive. Principles include speaking to the patient in a calm reassuring manner and avoiding repeated painful stimuli with close monitoring of vital signs.
\end{abstract}

Keywords: Coma, Depression, Dissociative Disorders, Oman, Pain.

\section{Introduction}

Coma is a deep state of prolonged unconsciousness in which a person fails to respond to painful stimuli, sounds or light. It is defined as a score of 8 or less on Glasgow Coma Scales (GCS). This scale is used to assess a person's level of consciousness after a head injury, but now it also clinically used in all acute medical and trauma patients.

The term coma is derived from Greek (kwup), meaning deep sleep. Moreover, general symptoms of comatose person are lack of response to painful or verbal stimuli, depressed brainstem reflexes, irregularity of breathing and absent of sleep-wake cycle. Coma may result from injury to cerebral cortex or reticular activating system via stroke, cardiac arrest, poisoning or trauma. The first priority to manage comatose patient is stabilizing respiration and circulation system. The status of coma may last days, weeks or years, some patients may enter vegetative status. Psychogenic coma is defined as a state of unresponsiveness without organic causes. Other names for psychogenic coma are dissociative stupor, conversion coma and factitious unconsciousness. In this case, we are reporting a psychogenic coma in older adult with major depressive disorder and up to our knowledge, it is first case reported in Oman.

\section{Case Report}

A 72-year-old male, was brought to Al Masarra Hospital Triage (AMH-ER) with a second episode of severe depressive episode with psychotic symptoms which were associated with suicidal ideation, but there was no clear plan to end his life. His condition started gradually deteriorating following cerebrovascular stroke five years ago, he exhibited depressed mood, loss of interest for any pleasurable activities almost every day and almost all over the day. The condition was associated with insomnia, poor appetite, easily provoked and social isolation. At that time, he sought psychiatric advice and was started on risperidone and mirtazapine, in which he showed improvement up to two months back before presenting to AMH-ER. Subsequently, his symptoms worsened and he started to exhibit nihilistic delusion as he believed he is going to die like his wife "who is already dead" in his mind. Also, he believed that he has no stomach and heart.

He presented to (AMH-ER) with florid psychotic symptoms which were associated with 
depressed mood, refusing food, insomnia and suicidal ideation. These symptoms indicated hospital admission and at that time memory impairment could not be ruled out due to severe depressive symptoms, no aggression or homicidal intention and no other psychotic symptoms were reported. He was admitted under geriatric inpatient services. He is dependent in all activities of daily livings (ADLs) and instrumental daily livings (IDLs). Medically, he is known to have hypertension and dyslipidaemia. He was observed to have masked face with slowness in movement initiation. Accordingly, he was referred to neurologist, who ruled out Parkinson's disease or any other neurological disorder.

During admission he had a fall from bed with apparent loss of consciousness and he was not showing response neither to verbal nor painful stimuli. On the other hand, his vital signs were within normal limits and no abnormal movements were observed. The patient was assessed by the physician who examined him and did not detect any cardiac, respiratory abnormalities or focal neurological signs and he advised to refer the patient for further assessment by expert neurologist to investigate the possible aetiology of unresponsiveness. He was referred to an extended medical facility and was assessed by multidisciplinary team which was composed of an internist and neurologist.

His neurological examination upon presentation showed brisk pupillary response for light with preserved conjunctival, corneal and gage reflex. He has no spontaneous motor movements but with normal tone. The deep tendons reflexes were not affected for both upper and lower limbs and on both sides. Babiniski's sign was equivocal. Eye examination revealed that patient was purposefully moving his pupils to avoid light. Doll's eye manoeuvre showed a response which was inconsistent with any neurological disease.

Metabolic work up done included complete blood count, renal function test, thyroid function test, liver function test, inflammatory markers, random blood sugar, and glycosylated hemoglobin. The results of those tests were normal in relation to his age. ECG showed no abnormalities and correlated with his clinical presentation. Also, CT head was requested twice, the first one was done on the onset time during loss of consciousness [Fig.1]. Then it was repeated after one day. Both revealed no acute brain insult, age-related atrophic changes, bilateral old infarcts and left basal ganglia old lacunar infarction. Doppler study of the carotid system was requested and it was also within normal limits [Fig.2] and chest CT showed changes which were suggesting asbestosis, he was assessed by chest medicine and reported as benign findings. No epileptiform activities were reported in his EEG. Three hours after he regained consciousness spontaneously and was referred back to Al Massarra hospital with impression of psychogenic coma after rolling out all possible organic etiology, than he continued his care under geriatric psychiatry unit for the main presenting symptoms and he was started on fluoxetine which tapered up to reach $40 \mathrm{mg}$ OD and olanzapine which tapered up to $10 \mathrm{mg} \mathrm{HS}$, however the
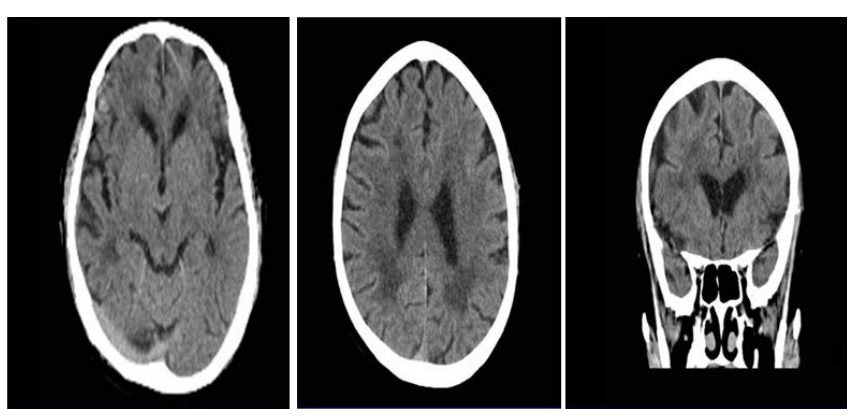

Fig.1: $C T$ head showed age-related atrophic changes, bilateral old infarcts and left basal ganglia old lacunar infarction.

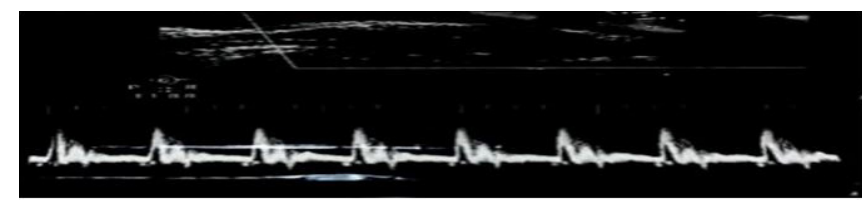

Fig.2: Doppler carotid showed normal findings. 
patient started to show slow and mild improvement concern his depressive symptomatology therefore the team decided to start ECT course after family consent and all pre ECT work up. After 12 sessions of ECT, the patient showed major improvement in his presentation. Following the improvements, he was referred to a clinical psychologist for assessment and further management as he was observed with memory impairment and cognitive slowness. A mild cognitive impairment (not related to ECT) was recorded after conducting cognitive assessment scales, also stress management were applied for both the patient and his family. He was discharged home and continued to follow-up in OPD.

\section{Discussion}

Unresponsive patient is one of the most worrisome presentations in medicine [1]. Coma is defined as lack of both awareness and response to external stimulation. However, as awareness is subjective and difficult to measure even in organic coma, apparent unconsciousness (usually motionless, with eyes closed) and unresponsiveness to external stimulation are more clinically useful defining features. The widely used Glasgow coma scale uses eye opening, motor and vocal response as proxy measures of vigilance and responsiveness [4]. Potential etiologies include cerebral hypoxia, intracranial hemorrhage, cerebrovascular accidents, drug effects to include over dosage and misadministration, and metabolic derangements [1].

Assessment of any patient in a state of coma, or unarousable unresponsiveness, includes a history taking and performing a general and focused neurologic examination. While the patient in a comatose state will be unable to provide a history, obtaining a description of the onset of coma and preceding events from witnesses and collateral history taking from reliable informant about the past medical, psychiatric, and drug history is essential. Review of medical notes often provides vital additional information. Finally, targeted investigations may be helpful both in terms of excluding important treatable causes of coma, and in making a positive diagnosis [2].

The case in this report, he had three hours course with acute onset of unresponsiveness during which he was vitally stable with accepted oxygen saturation level, ECG, lab investigations. CT scan revealed that no acute brain insult. Neurological examination by neurologist reported that no neurological deficits. However, during examination, the patient was tightly closing his eyes, eyelids are flickering, however, findings to support suppression of brain-stem function was minimal. This outcome could not be explained by medication-related, neurologic, or metabolic causes. On the basis of the medical and psychiatric assessments, we conclude that the delayed awakening was psychogenic.

Psychogenic coma was described in DSM IV as dissociative disorder NOS. however, it is omitted in DSM5. Although Plum and Posner in their presented data on 500 patients initially diagnosed with "coma of unknown etiology" only 8 of these patients were finally diagnosed with "psychiatric coma," $4(0.8 \%)$ of whom had a "conversion reaction" and with 2 cases each $(0.4 \%)$ of depression and catatonic stupor [5]. In a more recent study in 2189 patients treated over 8 years at an intensive care unit, only $0.3 \%$ were diagnosed with psychogenic coma, although this was a proportion of all coma and not just coma of initially unknown origin [6].

In psychoanalytic school, trauma during infancy and early childhood is recognized as a key factor leading to the emergence of dissociative processes, the potential dissociative structuring of the mind, and mind being characterized by multiple, discontinuous, centers of consciousness [2]. In our patient, it might be a dissociative reaction by the patient to perceived trauma or stress (symbolized of being mentally ill and admitted in mental hospital). A prompt diagnosis based on positive 
clinical signs may require some clinical experience but is essential to prevent unnecessary ongoing investigation and interventions with associated risks of iatrogenic harm, costs, and distress. Eye gaze test and hand drop test were positive. Patient with psychogenic coma avoids self-injury and hand falls to the side of rather than on to the patient's face (which occur in organic comma). What the positive signs of functional coma have in common is that they indicate (briefly) a normal voluntary nervous system that is inconsistent with the comatose presentation of the patient. The use of repeated painful or noxious stimuli is strongly contraindicated in psychogenic coma [3]. There may be severe psychological distress overwhelming the patient and it is important to remain sensitive in allowing the patient to gently recover.

Management of a coma due to dissociative disorder NOS is largely supportive. Principles include speaking to the patient in a calm reassuring manner and avoiding repeated painful stimuli with close monitoring of vital signs. Education of the family and other professionals that symptoms are real and the patient is not consciously feigning may be important. As profound psychological distress may underlie this disorder, there may be a shortterm role for medications such as anxiolytics or antipsychotics to assist the patient's return to full consciousness.

It will be valuable to re-add "psychogenic coma" specifically in the DSM 5 future modifications, including reliable epidemiological data, patterns of pre-existing and comorbid psychiatric illness, and the natural history and course of the illness including the long-term physical and psychological outcomes for these patients. Controlled studies of a variety of interventions (e.g., anxiolytics/ antipsychotics versus placebo, various interpersonal approaches to the patient) would be useful to determine whether these facilitate recovery; however, such studies would be difficult given the apparently very low incidence of the condition.

\section{Conclusion}

Management of a coma due to dissociative disorder NOS is largely supportive. Principles include speaking to the patient in a calm reassuring manner and avoiding repeated painful stimuli with close monitoring of vital signs. Education of the family and other professionals that symptoms are real and the patient is not consciously feigning may be important. As profound psychological distress may underlie this disorder, there may be a shortterm role for medications such as anxiolytics or antipsychotics to assist the patient's return to full consciousness.

Acknowledgments: Geriatric Team Members, Al Masarra Hospital, Oman.

Contributors: SAJ: manuscript writing, patient management; NAH: manuscript editing, patient management. SAJ will act as a study guarantor. Both authors approved the final version of this manuscript and are responsible for all aspects of this study.

Funding: None; Competing interests: None stated.

\section{References}

1. Downs JW, Young PE, Durning SJ. Psychogenic coma following upper endoscopy: A case report. Military Medicine. 2008;173:509.

2. Ludwig L, MCWhirter L, Willams S, Derry C, Stone J. Functional coma. In: Hallett M, Stone J, Carson AJ. Functional neurologic disorders, Handbook of Clinical Neurology, Vol. 139 ( $3^{\text {rd }}$ series). Elsevier. 2008; pp. 2-16.

3. Plum F, Posner JB. The diagnosis of stupor and coma. Contemp Neurol Ser. 1972;10:1-286.

4. Weiss N, Regard L, Vidal C, Luque Y, Taldir G, Vallet H, et al. Causes of coma and their evolution in the medical intensive care unit. J Neurol. 20212;259(7):1474-1477.

5. Itzkowitz S. Dissociative turn in psychoanalysis. Am J Psychoanal. 2015;75(2):145-153.

6. Jackson AO. Faking unconsciousness. Anaesthesia. 2000;55(4):409. 